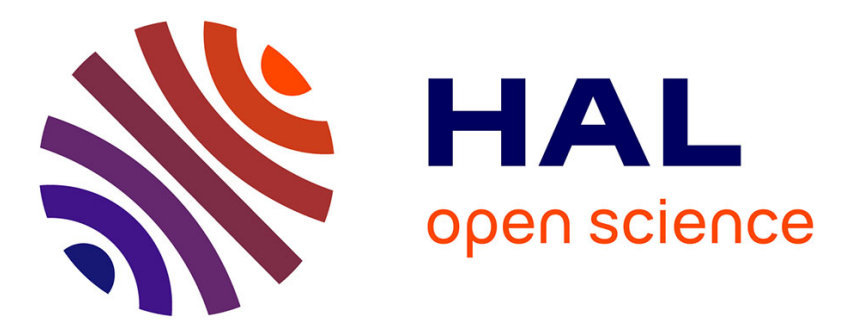

\title{
Impact of the spin state switching on the dielectric constant of iron (II) spin crossover nanoparticles
}

Antonio Iazzolino, Geoffrey Galle, Jérôme Degert, Jean François Létard, Eric

Freysz

\section{- To cite this version:}

Antonio Iazzolino, Geoffrey Galle, Jérôme Degert, Jean François Létard, Eric Freysz. Impact of the spin state switching on the dielectric constant of iron (II) spin crossover nanoparticles. Chemical Physics Letters, 2015, 641, pp.14-19. 10.1016/j.cplett.2015.10.038 . hal-01256753

\section{HAL Id: hal-01256753 \\ https://hal.science/hal-01256753}

Submitted on 15 Jan 2016

HAL is a multi-disciplinary open access archive for the deposit and dissemination of scientific research documents, whether they are published or not. The documents may come from teaching and research institutions in France or abroad, or from public or private research centers.
L'archive ouverte pluridisciplinaire HAL, est destinée au dépôt et à la diffusion de documents scientifiques de niveau recherche, publiés ou non, émanant des établissements d'enseignement et de recherche français ou étrangers, des laboratoires publics ou privés.

\section{다(1)(2)}

Distributed under a Creative Commons Attribution - ShareAlikel 4.0 International 


\title{
Impact of the spin state switching on the dielectric constant of iron (II) spin crossover nanoparticles
}

\author{
Antonio Iazzolino ${ }^{\mathrm{a}}$, Geoffrey Galle ${ }^{\mathrm{a}}$, Jérôme Degert ${ }^{\mathrm{a}}$, Jean François Létard ${ }^{\mathrm{b}}$, Eric Freysz ${ }^{\mathrm{a}, *}$ \\ a LOMA, Université de Bordeaux, UMR CNRS 5798, 351 cours de la libération, 33405 Talence Cedex, France \\ b OliKrom, 16 Avenue Pey Berland, bâtiment ChemInnov, 33607 Pessac, France
}

\section{A B S T R A C T}

A simple model makes it possible to relate the dielectric constant of spin-crossover nanoparticles to the indices of refraction and the absorption coefficients of solutions containing such nanoparticles. Using this model we show that in the visible spectral range, the switching from a diamagnetic (low spin state) to a paramagnetic (high spin state) state results in a noticeable change of the dielectric constants of the spin crossover nanoparticles containing 3,5 and 7 iron (II) ions. Within the studied temperature range and for all the studied nanoparticles, the variation of their index of refraction is about $10 \%$.

Media whose optical dielectric constant can be tuned by different means open a wide range of possibilities in the design of optical or data storage components [1]. Among such media, optically bistable dispersions of $\mathrm{Fe}^{\mathrm{II}}$ spin-crossover nanoparticles (SCO NPs) are very promising [2-12]. Some of these SCO NPs present a large thermal hysteresis loop at room temperature. Within the hysteresis loop, the magnetic state of these NPs can be reversibly switched from a diamagnetic low spin $(L S, S=0)$ state toward a paramagnetic high spin $(\mathrm{HS}, S=2)$ state. This spin state transition is due to electron spin pairing $(S=0)$ or unpairing $(S=2)$ within the $\mathrm{Fe}^{\mathrm{II}}$ ions. At the macroscopic scale the spin transition is associated with a phase transition. This phase transition can be induced by many means using thermal or pressure variations, laser or magnetic excitations. For most of the SCO NPs, the spin state transition results in marked absorption changes. For instance, a solution containing SCO NPs is almost uncolored when the particles are in the HS state and turns red or pink when the NPs are in the LS state. This pink color is related to a broad absorption band at around $520 \mathrm{~nm}$, which is characteristic of the $\mathrm{d}-\mathrm{d}$ transition of the Fe $\mathrm{Fe}^{\mathrm{II}}$ ions in the LS state $\left({ }^{1} \mathrm{~A}_{1 \mathrm{~g}} \rightarrow{ }^{1} \mathrm{~T}_{1 \mathrm{~g}}\right)$ [13]. The $\mathrm{d}-\mathrm{d}$ transition of the $\mathrm{Fe}^{\mathrm{II}}$ ions in HS state $\left({ }^{5} \mathrm{~T}_{2 \mathrm{~g}} \rightarrow{ }^{5} \mathrm{E}_{\mathrm{g}}\right)$ is expected at lower energy in the near infrared region $(\sim 830 \mathrm{~nm})$ [13]. This accounts for the absence of color of the solution containing the SCO NPs in the HS state. SCO NPs with a broad thermal hysteresis loop at room temperature and large absorption changes are therefore very good candidates for data storage. Indeed as previously shown, the spin state transition results in a change in the dielectric constant of the SCO NPs [14-19]. This should result in

\footnotetext{
* Corresponding author.

E-mail address: eric.freysz@u-bordeaux.fr (E. Freysz).
}

both a change in the absorption coefficient and the index of refraction. While the change in the absorption of SCO NPs during a spin state transition can be easily monitored, very little is known about the evolution of their index of refraction [20]. Different experiments have been dedicated to the investigation of the evolution of the dielectric properties of SCO bulk materials [14-19]. However up-to now and to the best of our knowledge, the evolution of the dielectric constant associated with spin transition of SCO NPs in the UV, visible and near IR spectral range has been barely investigated [21]. Hereafter, we propose a simple technique which makes it possible to determine the optical dielectric constant of a solution of SCO NPs. A simple model is used to compute the dielectric constant of the SCO NPs and their evolution versus temperature and high spin fraction. While over the studied temperature we demonstrate that spin state transition can result in variation of the relative change of the index of refraction $(\Delta n / n)$ that can be as high as $7 \%$. Such SCO NPs can be used to adjust on demand (or 'tune') the optical dielectric constant of a given dielectric material.

The selected SCO complex belongs to the $\left[\mathrm{FeL} \alpha(X)_{2}\right]$ family (with $\mathrm{L}=\mathrm{NH}_{2}$-trz(4-amino-1,2,4-triazole) and $X$ is $\mathrm{Br}$ anion). In bulk, it is known to display interesting magnetic properties with a spin transition at room temperature. The synthesis of NPs and the properties of such compounds have already been published $[5,6]$. To synthesize the SCO NPs we used reverse micelle techniques. A reverse micelle is a dispersion of polar liquid drops coated with surfactant molecules, a few nanometers in diameter in suspension in a continuous oil phase. The associated solution is called a microemulsion. A microemulsion is therefore homogeneous at the macroscopic scale but the non-miscible components are separated at the microscopic scale. Furthermore, the different micelles exchange their different constituents very rapidly. In our case, the oil-phase is 

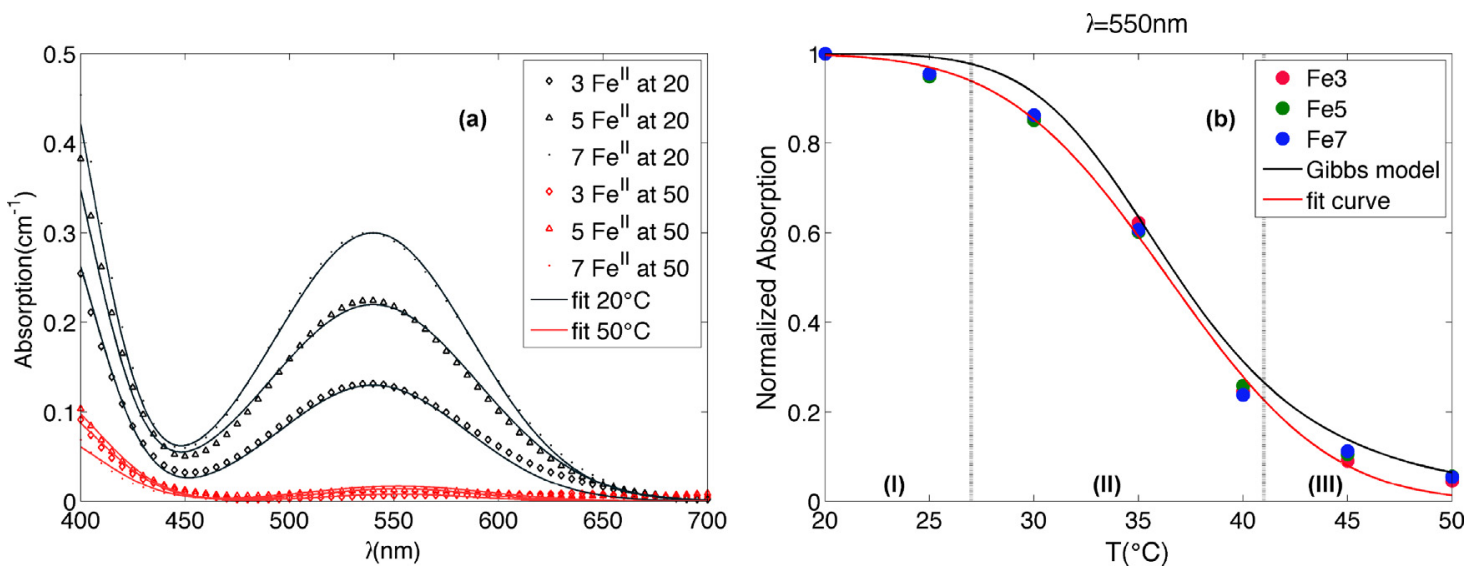

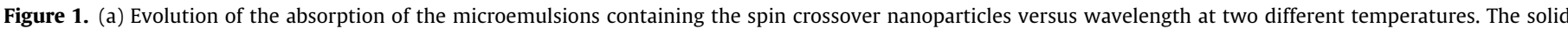

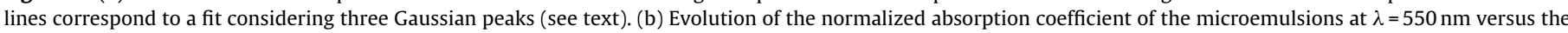

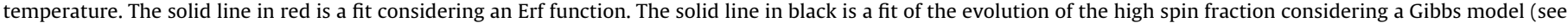
text; For interpretation of the references to colour in this figure legend, the reader is referred to the web version of this article.).

heptane, the polar phase is water and the surfactant is AOT (dioctyl sulfosuccinate sodium salt). To synthesize the SCO NPs, two different microemulsions were prepared by dissolving in demineralized water iron (II) salt $\mathrm{FeBr}_{2}$ and aminotriazole, respectively. The other constituents were identical. The volume fraction of micelles in the microemulsions is about $3 \%$. Once prepared, the two microemulsions were mixed and stirred for a few minutes. The ratio between the iron (II) salt and aminotriazole within the micelles makes it possible to control the size of synthesized SCO NPs. NPs containing 3, 5 or 7 iron (II) anions have been prepared. The length of the associated SCO NPs is about $0.77 \mathrm{~nm}, 1.53 \mathrm{~nm}, 2.3 \mathrm{~nm}$, respectively. These sizes have to be compared to the radius of the micelles. According to the dynamic light scattering experiment we performed, it is $\sim 3 \mathrm{~nm}$. We noticed that for more than 7 iron (II) ions the solution turns into a gel suggesting a destabilization of the microemulsion by the SCO NPs.

The measurements of the absorption coefficient $\alpha$ of the different solutions of SCO NPs were performed at different temperatures using a Shimadzu UV-VIS-NIR spectrophotometer (UV-3600). The main advantages of the as prepared solutions is that, due to the small size of the micelles and NPs, only absorption and Rayleigh diffusion account for the attenuation of the visible light. This is of particular interest for many applications where transparency of the material in the optical range is required. Figure 1 displays the evolution of the absorbance of solutions of NPs containing 3, 5 and 7 $\mathrm{Fe}^{\mathrm{II}}$ ions in the $400-700 \mathrm{~nm}$ spectral range for different temperatures. At room temperature, the microemulsion is pink and a weak absorption band centered at $550 \mathrm{~nm}$ is recorded. The peak of this absorption band increases linearly with the number of $\mathrm{Fe}^{\mathrm{II}}$ ions in the microemulsion. As we previously mentioned, this absorption peak is associated with the $\mathrm{d}-\mathrm{d}$ transition of the $\mathrm{Fe}^{\mathrm{II}}$ ions in the LS state. The solution also presents strong absorption peaks centered at $380 \mathrm{~nm}$ and $280 \mathrm{~nm}$ that are associated to metal-ligand charge transfer (MLCT) and ligand-metal charge transfer (LMCT) bands (not presented here). The absorption band centered at $380 \mathrm{~nm}$ is responsible for the absorption shoulder we recorded at $400 \mathrm{~nm}$, displayed in Figure 1a. We were able to accurately fit (see Figure 1a) the absorption coefficient of the solutions considering three Gaussian absorption peaks centered at $550 \mathrm{~nm}, 380 \mathrm{~nm}$ and $280 \mathrm{~nm}$, respectively. This indicates that the contribution of Rayleigh diffusion to the attenuation of light beam in these solutions is negligible. When the temperature of the solution is increased above $40^{\circ} \mathrm{C}$, the $\mathrm{d}-\mathrm{d}$ transition of the $\mathrm{Fe}^{\mathrm{II}}$ ions is shifted toward the infrared and the MLCT absorption bands strongly weaken. As a consequence, the microemulsion becomes transparent in the visible range. At $50^{\circ} \mathrm{C}$, the absorbance of the solution at $550 \mathrm{~nm}$ is reduced by an order of magnitude. Here again, we were able to accurately fit the absorption in the visible range, considering that the solution has only strong absorption band centered at $280 \mathrm{~nm}$ and $380 \mathrm{~nm}$.

To measure the evolution of the index of refraction of the different microemulsions, they were inserted in a glass prism with an apex angle $(A)$ of $45^{\circ}$. The temperature of the prism was finely controlled, and the minimum angle of deviation, $D(\lambda)$, for the prism filled with the microemulsions was measured at different wavelengths in the visible range. The latter, which depends on the apex angle $A$, is related to the index of refraction index $n(\lambda)$ of the microemulsion at a given wavelength $\lambda$ by:

$n(\lambda)=\frac{\sin ((D(\lambda)+A) / 2)}{\sin (A / 2)}$

Our experimental set-up makes it possible to measure the index of refraction of a solution with a precision better that $5 \times 10^{-4}$. The evolution of the refractive index of n-heptane and microemulsions containing 3, 5 and $7 \mathrm{Fe}^{\mathrm{II}}$ ions at $20^{\circ} \mathrm{C}$ and $50^{\circ} \mathrm{C}$ is displayed in Figure 2.

As expected, the index of n-heptane decreases with temperature and wavelength. The index of the microemulsions doped with SCO NPs follows the same trend. The steady decrease of the index versus the wavelength indicates that the dispersion of the doped microemulsion is mainly related to the dispersion of n-heptane. However, one can notice that whatever the temperature or the wavelength, the index of the doped solution increases. This indicates that the index of refraction of the solution is impacted by the presence of the SCO NPs. The insertion of SCO NPs within the micelles modifies the real $\varepsilon^{\prime}$ and imaginary $\varepsilon^{\prime \prime}$ part of the dielectric constant $\varepsilon$ of the microemulsion. The latter are related to the index of refraction $n$ and the absorption coefficient $\alpha$ through the following relations: $\varepsilon^{\prime}=n^{2}-k^{2}$ and $\varepsilon^{\prime \prime}=2 n k$, where $k=\lambda \alpha / 4 \pi$. Hence, one may consider retrieving the index of the SCO NPs knowing the initial and modified dielectric constants of the host medium. Reynolds and Hough have overviewed the different models which have been proposed to compute the dielectric constant of a dielectric medium doped with nanoparticles of different shape [22]. Hereafter we use the model proposed by Landau [23]. It relates the dielectric constant $\varepsilon_{M}$ of the doped medium to the dielectric constant of the host medium $\varepsilon_{1}$ homogeneously doped with dielectric nanoparticles 

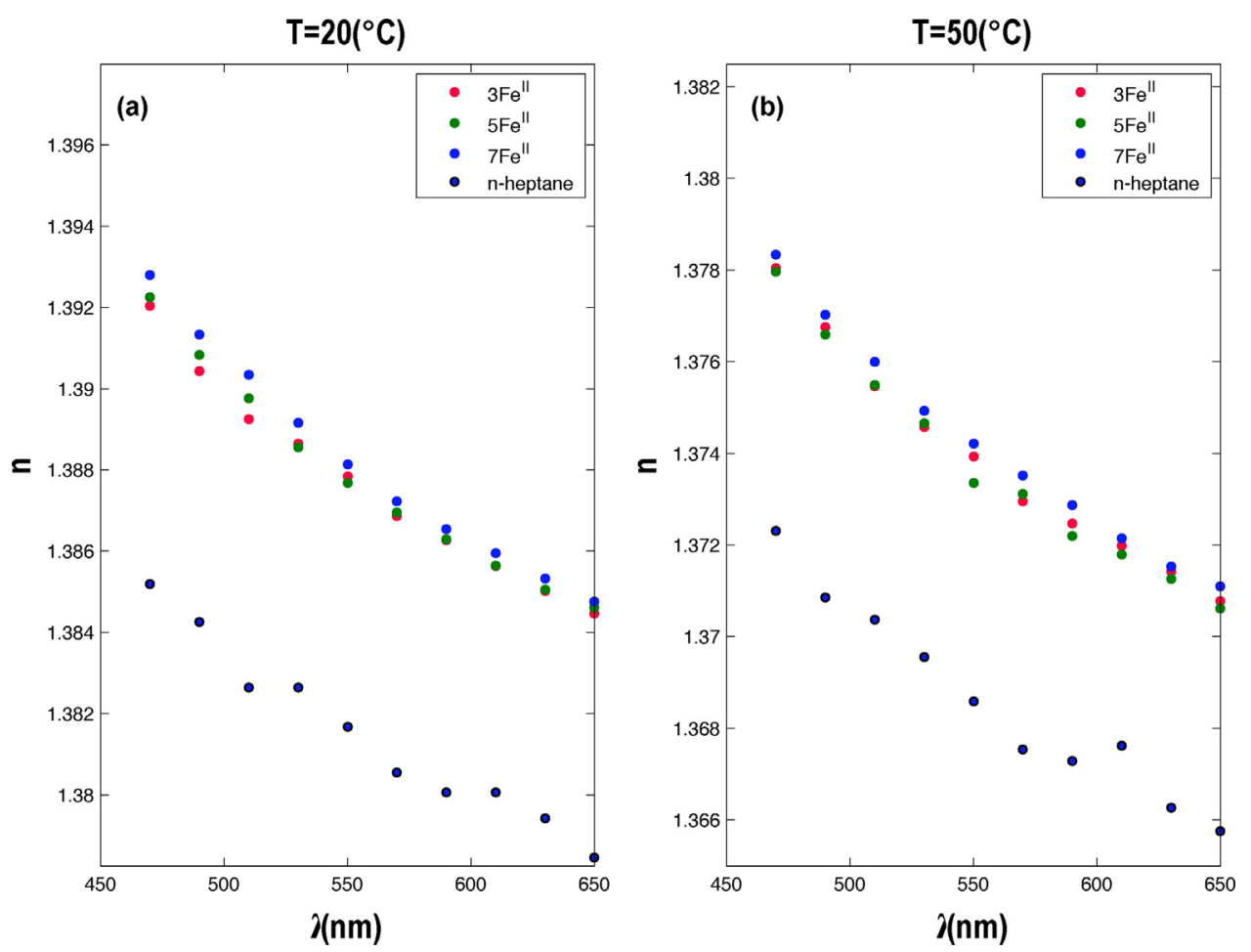

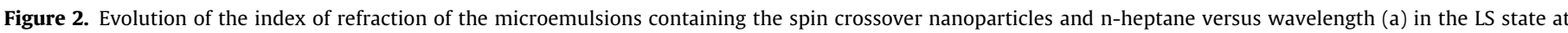
$T=20^{\circ} \mathrm{C}$, (b) in the HS state at $T=50^{\circ} \mathrm{C}$

with the dielectric constant $\varepsilon_{2}$ whose size is small compared to the optical wavelength and it gives:

$\varepsilon_{M}=\varepsilon_{1}+\frac{c\left(\varepsilon_{1}-\varepsilon_{2}\right) \varepsilon_{1}}{2 \varepsilon_{1}+\varepsilon_{2}}$

where $c$ is the volume fraction of the NPs. This model is valid up to a volume fraction of $10 \%$ and therefore valid for our solutions containing $3 \%$ of doped micelles. Hereafter we will consider that within the studied spectral range the dielectric constant of the host medium (i.e. n-heptane) is real (i.e. $\left.\varepsilon_{1}=\left(n_{\text {heptane }}\right)^{2}\right)$ whereas the dielectric constant $\varepsilon_{2}$ of the NPs is a complex number with real $\left(\varepsilon_{2}^{\prime}\right)$ and an imaginary $\left(\varepsilon_{2}^{\prime \prime}\right)$ part. As indicated by Eq. (1), knowing the concentration $c$ of NPs, the dielectric constant of the host (i.e. $\varepsilon_{1}$ ) and doped host medium (i.e. $\varepsilon_{M}$ ), one can compute the dielectric constant of the SCO NPs (i.e. $\varepsilon_{2}$ ). As displayed in Figure 3, this procedure makes-it possible to compute the evolution of the index $n_{2}$ and the attenuation coefficient $k_{2}$ associated with the dielectric constant of the doped micelles containing 3, 5 and $7 \mathrm{Fe}^{\mathrm{II}}$ ions. At $\lambda=510 \mathrm{~nm}$ and at room temperature, the index of refraction of the micelles $n_{2}$ (LS) containing 3,5 and $7 \mathrm{Fe}^{\mathrm{II}}$ ions is $1.61,1.63,1.65$, respectively (Figure 3c). These values have to be compared to the index of refraction of the doped micellar solutions: $n \sim 1.39$ (see Figure $2 \mathrm{a}$ ). Moreover and as expected, the absorption peak of the micelles centered at $550 \mathrm{~nm}$ increases almost linearly with the number of $\mathrm{Fe}^{\mathrm{II}}$ ions within the micelles (Figure $3 \mathrm{a}$ ). At $50^{\circ} \mathrm{C}$, the absorption of the micelles is reduced (Figure $3 \mathrm{~b}$ ). The remaining absorption band centered at $550 \mathrm{~nm}$ indicates that some of the $\mathrm{Fe}^{\mathrm{II}}$ ions in the micelles are still in the HS state. The index of refraction $n_{2}(\mathrm{HS})$ of the micelles containing 3, 5 and $7 \mathrm{Fe}^{\mathrm{II}}$ ions decreases to about 1.54, 1.54 and 1.56 (Figure 3d). These values have to be compared to the index of refraction of the doped micellar solutions: $n \sim 1.375$ (see Figure $2 b$ ). One can also notice that the LS to HS state transition results in a relative index change defined as $\left(n_{2}(\mathrm{LS})-n_{2}(\mathrm{LS})\right) / n_{2}(\mathrm{HS}) \sim 4.5 \%, 5.8 \%$ and $5.8 \%$ for 3, 5 and $7 \mathrm{Fe}^{\mathrm{II}}$ ions in the micelles.

So far, we have computed the index and absorption of the micelles containing the SCO NPs. To further proceed and to calculate the index of the SCO NPs, we have considered that the micelles containing the NPs are similar to core-shell nanoparticles (CS-NPs). The core of the CS-NP is the SCO NPs and has a dielectric constant $\varepsilon_{C}$. The shell of the CS-NP is made out of water and surfactant molecules characterized as a whole by a dielectric constant $\varepsilon_{s}$. To further simplify the extraction of the index of the SCO NPs, we consider that the CS-NP and SCO NPs have a spherical shape. The dielectric constant $\varepsilon_{C S}$ of such CS NPs is [24,25]:

$\varepsilon_{C S}=\varepsilon_{S}+\frac{\rho\left(\varepsilon_{C}+2 \varepsilon_{S}\right)+2\left(\varepsilon_{C}-\varepsilon_{S}\right)}{\rho\left(\varepsilon_{C}+2 \varepsilon_{S}\right)-\left(\varepsilon_{C}-\varepsilon_{S}\right)}$

where $\rho$ is the ratio between the volume of the shell i.e. $4 \pi\left(a^{3}-b^{3}\right) / 3$ and the core i.e. $\left(4 \pi b^{3} / 3\right)$, respectively. As already mentioned, the micelles, coated with AOT surfactant molecules whose length is $\sim 0.15 \mathrm{~nm}$, have a radius $a \sim 3 \mathrm{~nm}$. Hence hereafter, we will neglect the contribution to the index of this shell of surfactant molecules whose index of refraction in the visible range is $\sim 1.5$. The main constituent of the shell of the micelles is thus water whose dielectric constant $\varepsilon_{S}$ is well defined in the visible range. The effective radius b of the SCO NPs is about $0.7 \mathrm{~nm}, 1.6 \mathrm{~nm}$ and $2.3 \mathrm{~nm}$ for NPs containing 3, 5 and $7 \mathrm{Fe}^{\mathrm{II}}$ ions, respectively. According to Eq. (2), knowing the dielectric constant $\varepsilon_{C S}$ of the CS-NPs, the dielectric constant of the shell $\varepsilon_{S}$ and the ratio $\rho$, one is able to compute the dielectric constant $\varepsilon_{C}$ of the SCO NPs. The evolution of index $n_{C}$ and absorption constant $k_{C}$ for SCO NP containing 3, 5 and $7 \mathrm{Fe}^{\mathrm{II}}$ ions in the LS and HS state is displayed in Figure 4. As the SCO NPs are switched from the LS to HS state, $k_{C}$ is reduced by almost one order of magnitude. At $T=20^{\circ} \mathrm{C}$ and at $\lambda=510 \mathrm{~nm}$, the index of refraction of the SCO NP is $\sim 1.71,1.74$ and 1.76 for NP with 3 , 5 and $7 \mathrm{Fe}^{\mathrm{II}}$ ions (Figure 4c). The latter are about 1.57, 1.58, 1.63 at $50^{\circ} \mathrm{C}$. Hence the switching from the LS to HS state results in a very large relative index change (i.e. $\left.\left(n_{H S}-n_{L S}\right) / n_{H S}\right)$ that is on average $\sim 10 \%$.

However, the change of the index of refraction $\Delta n_{\lambda}=n_{\lambda, H S}-n_{\lambda, L S}$ of the SCO NPs versus the wavelength $\lambda$ 


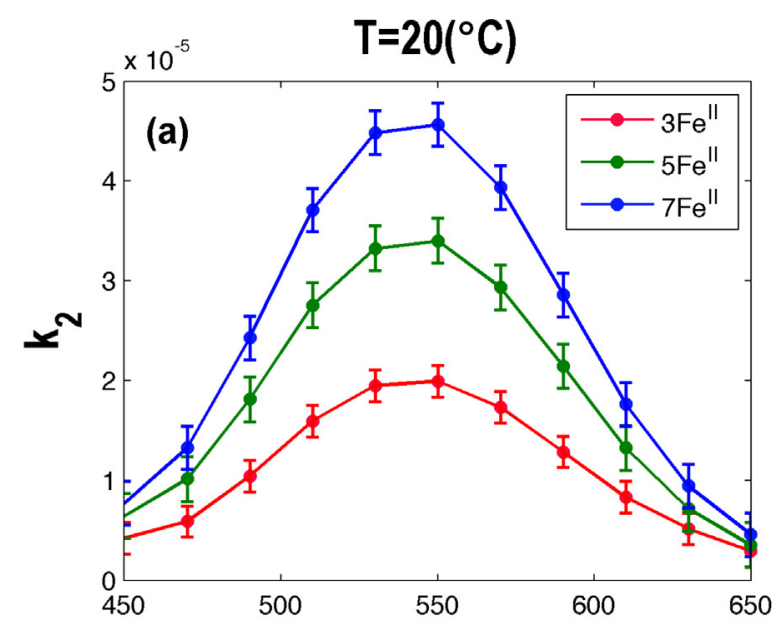

$\mathrm{T}=20\left({ }^{\circ} \mathrm{C}\right)$

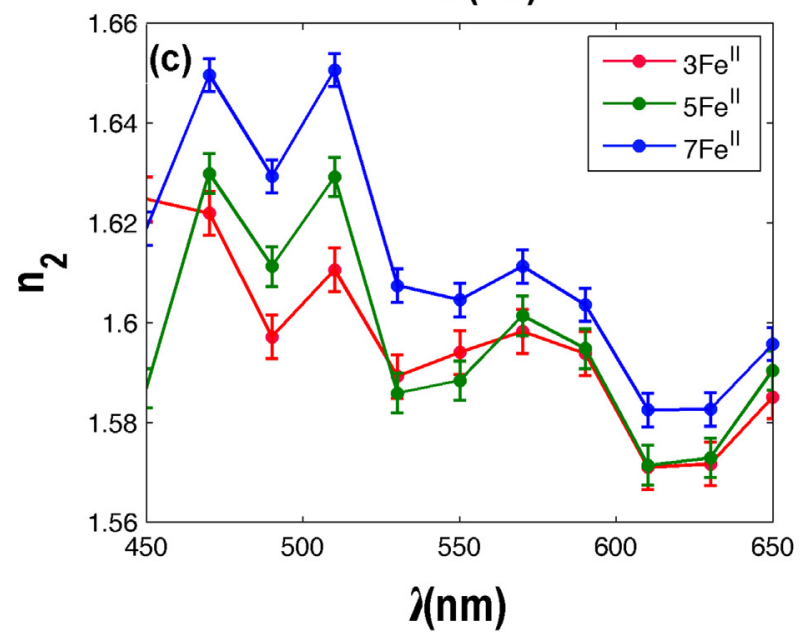

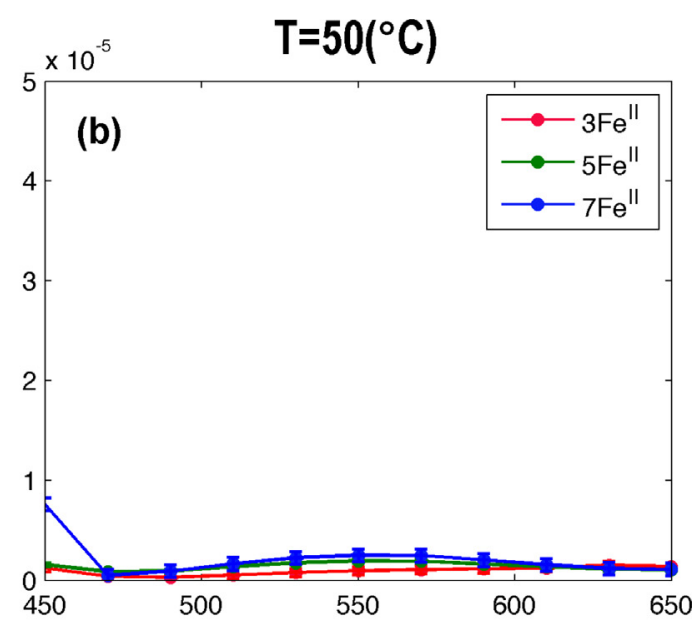

$\mathrm{T}=50\left({ }^{\circ} \mathrm{C}\right)$

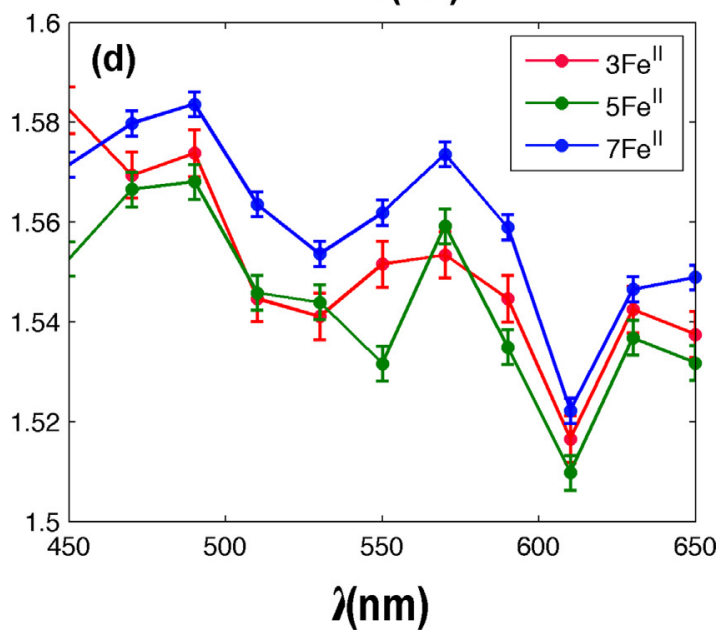

Figure 3. Evolution of the absorption and the index of the micelles containing the spin crossover nanoparticles versus the wavelength at two different temperatures.

displayed in Figure 4 results from both the variation of the temperature $\Delta T$ and the HS spin fraction $\Delta \gamma$. To the first order and over a small range of temperature variation, it can be approximated by:

$\Delta n_{\lambda}(T, \gamma)=\left(\frac{\partial n}{\partial T}\right)_{\lambda} \Delta T+\left(\frac{\partial n}{\partial \gamma}\right)_{\lambda} \Delta \gamma$

We firstly evaluated the evolution of $\gamma(T)$ from the normalized absorption coefficient (Figure $1 \mathrm{~b}$ ) at $\lambda=550 \mathrm{~nm}$. Indeed the latter quantity scales like the LS fraction which is equal to $1-\gamma$. Actually, from thermodynamical consideration and according to a Gibbs model, $\gamma(T)$ scales like:

$\gamma(T)=\frac{1}{1+\exp \left[\frac{\Delta H}{R}\left(\frac{1}{T}-\frac{1}{T_{C}}\right)\right]}$

where $\Delta H$ and $T_{c}$ are the spin transition enthalpy and the critical temperature for which $\gamma=0.5$, respectively. According to our best fitting, we found $T_{c}=35^{\circ} \mathrm{C}$ and $\triangle H \sim 37 \mathrm{~kJ} \mathrm{~mol}^{-1}$ (see Figure $1 \mathrm{~b}$ ). The latter is in good agreement with enthalpy values usually reported for SCO compounds. For more accurate determination of $(\partial n / \partial T) \lambda$ and $(\partial n / \partial \gamma) \lambda$, we also fitted $\gamma(T)$ with an Erf function. Although the latter fitting is less physical, it fits more accurately our experimental data (see Figure 1b). We also split the temperature in three domains (see Figure 1b). The first one, where the SCO NPs are almost in the LS state (i.e. $\gamma<0.1$ ), lies in between $T=20^{\circ} \mathrm{C}$ and $T=27^{\circ} \mathrm{C}$. The second one where the SCO NPs are switching from LS to HS state, lies in between $T=27^{\circ} \mathrm{C}$ and $T=42^{\circ} \mathrm{C}$. And finally, the third domain where the spin transition is almost over (i.e. $\gamma>0.9$ ), lies above $T=42^{\circ} \mathrm{C}$. In the three domains, we evaluated the impact of the temperature variation on the index variation of the SCO NPs. The value of these coefficients at $\lambda=550 \mathrm{~nm}$ is reported in Table 1 .

In Table 1 , note that the value deduced in region III are at least one order of magnitudes smaller than the ones found in regions I and II. They should be considered as irrelevant due to our experimental uncertainties. As expected in conventional materials, Table 1 indicates that $\partial n / \partial T$ is negative. However, it is found to decrease as the temperature increase. Table 1 also indicates that $\partial n / \partial \gamma$ is positive and points out that the index of refraction decreases as their HS fraction increases. The latter coefficient makes it possible to evaluate the sole contribution to

Table 1

Evolution of $(\partial n / \partial T)_{\lambda}$ and $(\partial n / \partial \gamma)_{\lambda}$.

\begin{tabular}{llllr}
\hline$\lambda=550 \mathrm{~nm}$ & Domain & $3 \mathrm{Fe}^{\mathrm{II}}$ & $5 \mathrm{Fe}^{\mathrm{II}}$ & \multicolumn{1}{c}{$7 \mathrm{Fe}^{\mathrm{II}}$} \\
\hline$\frac{\partial n}{\partial T}\left({ }^{\circ} \mathrm{C}^{-1}\right)$ & I & -0.00118 & -0.0121 & -0.0093 \\
& II & -0.0081 & -0.0102 & -0.0055 \\
& III & -0.0001 & -0.0012 & 0.0004 \\
$\frac{\partial n}{\partial \gamma}$ & & & & \\
& I & 0.3128 & 0.261 & 0.2735 \\
& II & 0.1074 & 0.1167 & 0.0815 \\
& III & -0.0012 & -0.0176 & -0.0068 \\
\hline
\end{tabular}




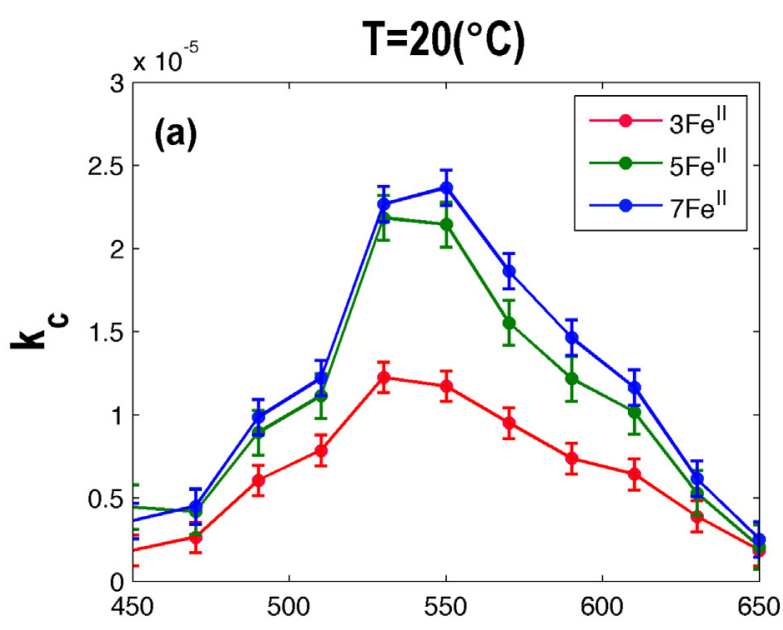

$\mathrm{T}=20\left({ }^{\circ} \mathrm{C}\right)$

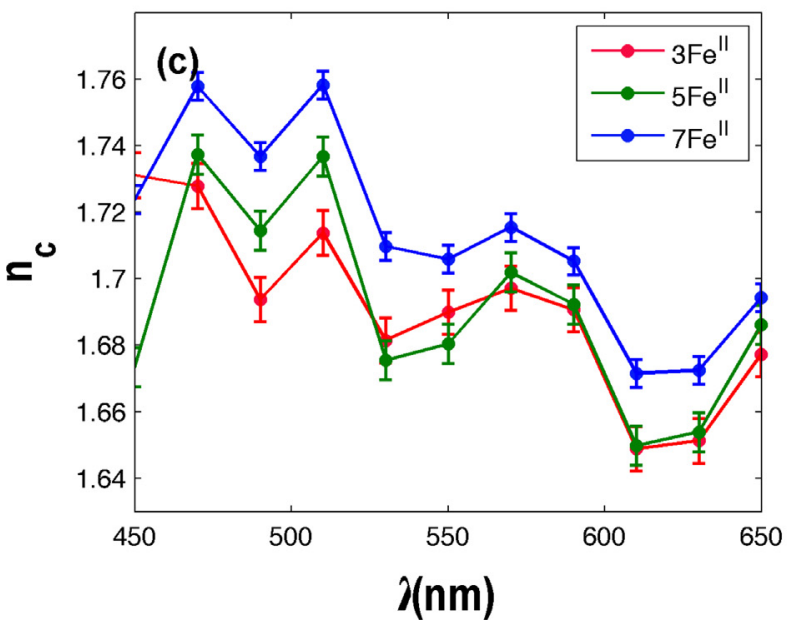

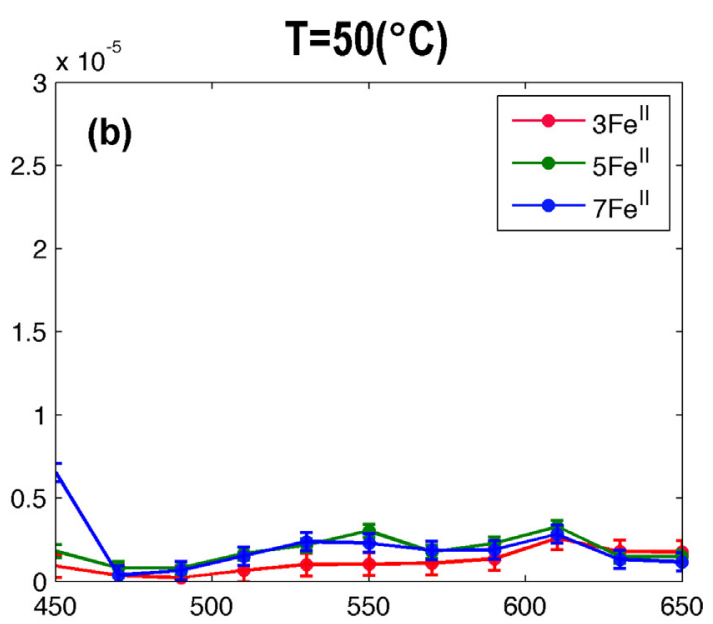

$\mathrm{T}=50\left({ }^{\circ} \mathrm{C}\right)$

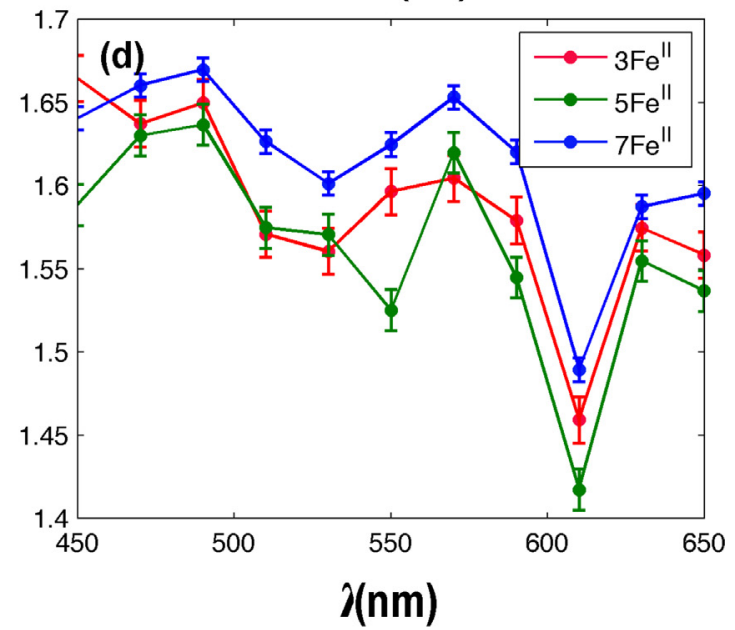

Figure 4. Evolution of the absorption and the index of the spin crossover nanoparticles versus wavelength at two different temperatures.

the index of refraction of the SCO NPs associated to the switching from the LS to the HS state. It is $\Delta n \sim 0.12,0.12$ and 0.10 for 3, 5 and $7 \mathrm{Fe}^{\mathrm{II}}$ ions, respectively. The latter values indicate that the index change due to the LS-HS switching is large and $\triangle n / n$ is $\sim 7 \%$ for all the studied SCO NPs. This stresses the interest of SCO compounds with steep thermal transition or sharp thermal hysteresis loop in the optical domain. However, within our experimental uncertainties and surprisingly, one cannot clearly evidence the impact of the numbers Fe ${ }^{\mathrm{II}}$ ions within the SCO NPs on $\partial n / \partial T$ and $\partial n / \partial \gamma$. Finally, we should mention that one may also expect to observe a dispersion of the index of refraction of the SCO NPs across the absorption peak centered at $550 \mathrm{~nm}$. However, the latter dispersion is usually small when the absorption peak is broad.

In conclusion, we have developed a method that makes it possible to measure the dielectric constant of thermally switchable SCO NPs. This technique has been applied to follow the evolution of the dielectric constant of Fe ${ }^{\mathrm{II}}$ NPs during their thermal switching. Our measurements indicate the switching from the LS to the HS state of these SCO NPs results in a large change of the real and imaginary part of their dielectric constants. In the visible range and for the temperature in between $20^{\circ} \mathrm{C}$ to $50^{\circ} \mathrm{C}$, the index change $\Delta n / n$ is evaluated to be as large as $12 \%$. Our technique also makes it possible to evaluate the individual contributions of the temperature and the magnetic state to the variation in the index of refraction.

\section{Acknowledgments}

The authors acknowledge supports through ANR project RMNP 019-01 'OptoMaTS,' Conseil Régional d'Aquitaine via the projects 'MatTS and Isocel,' and the ADEME through the project 'Isocel'.

\section{References}

[1] O. Kahn, J.C. Martinez, Science 279 (1998) 44-48.

[2] E. Coronado, J.R. Galán-Mascarós, M. Monrabal-Capilla, J. García-Martínez, P. Pardo-Ibáñez, Adv. Mater. 19 (10) (2007) 1359.

[3] J.R. Galán-Mascarós, E. Coronado, A. Forment-Aliaga, M. Monrabal-Capilla, E. Pinilla-Cienfuegos, M. Ceolin, Inorg. Chem. 49 (12)(2010) 5706.

[4] J.-F. Létard, P. Guionneau, L. Goux-Capes, Top. Curr. Chem. 235 (2004) 221.

[5] T. Forestier, S. Mornet, N. Daro, T. Nishihara, S.-I. Mouri, K. Tanaka, O. Fouche, E. Freysz, J.-F. Létard, Chem. Commun. (2008) 4327.

[6] T. Forestier, A. Kaiba, S. Pechev, D. Denux, P. Guionneau, C. Etrillard, N. Daro, E. Freysz, J.F. Létard, Chem. Eur. J. 15 (2009) 6122.

[7] E. Coronado, J.R. Galán-Mascarós, M. Monrabal-Capilla, J. Garciía-Martínez, P. Pardo-Ibánez, Adv. Mater. 19 (2007) 1359.

[8] J.R. Galan-Mascaros, E. Coronado, A. Forment-Aliaga, M. Monrabal-Capilla, E. Pinilla-Cienfuegos, M. Ceolin, Inorg. Chem. 49 (2010) 5706.

[9] A. Tokarev, L. Salmon, Y. Guari, W. Nicolazzi, G. Molnár, A. Bousseksou, Chem. Commun. 46 (2010) 8011.

[10] I. Boldog, A.B. Gaspar, V. Martnez, P. Pardo-Ibanez, V. Ksenofontov, A. Battacharjee, P. Gutlich, J.A. Real, Angew. Chem. Int. Ed. 47 (2008) 6433.

[11] F. Volatron, L. Catala, E. Rivière, A. Gloter, O. Stephan, T. Mallah, Inorg. Chem. 47 (2008) 6584.

[12] C. Shi, X. Zhang, Y. Cai, Y. Yao, W. Zhang, Angew. Chem. Int. Ed. 54 (2015) 6206

[13] A. Hauser, Top. Curr. Chem. 233 (2004) 49.

[14] A. Hauser, Chem. Phys. Lett. 202 (1993) 173. 
[15] A. Bousseksou, G. Molnar, P. Demont, J. Menegotto, J. Mater. Chem. 13 (2003) 2069.

[16] S. Bonhommeau, T. Guillon, L.M.L. Daku, P. Demont, J.S. Costa, J.-F. Letard, G. Molnar, A. Bousseksou, Angew. Chem. Int. Ed. 45 (2006) 1625.

[17] P. Mounaix, N. Lascoux, J. Degert, E. Freysz, A. Kobayashi, N. Daro, J.-F. Létard, Appl. Phys. Lett. 89 (2006) 174105

[18] B. Viquerat, G. Degert, C. Mauriac, J.F. Létard, E. Freysz, Appl. Phys. Lett. 99(2011) 061908.

[19] E.D. Loutete-Dangui, F. Varret, E. Codjovi, P.R. Dahoo, H. Tokoro, S. Ohkoshi, C. Eypert, J.-F. Létard, J.M. Coanga, K. Boukheddaden, Phys. Rev. B 75 (2007) 184425.
[20] C. Arnaud, T. Forestier, N. Daro, E. Freysz, J.F. Létard, G. Pauliat, G. Roosen, Chem. Phys. Lett. 470 (2009) 131.

[21] C. Lefter, I.A. Gural'skiy, H. Peng, G. Molnár, L. Salmon, A. Rotaru, A. Bousseksou, P. Demont, Physica Status Solidi (RRL) 8 (2) (2014) 192

[22] J.A. Reynolds, J.M. Hough, Proc. Phys. Soc. B 70 (1957) 769

[23] L. Landau, E. Lifchitz, Electrodynamique des Milieux Continus, Edition Mir Moscou, 1969, pp. 70, Chapter 2.

[24] C.F. Bohren, D.R. Huffman, Absorption and Scattering of Light by Small Particles, Wiley-VCH Verlag GmbH, 2007, pp. 137, Chapter 5.

[25] K.C. Uday, N. Engheta, Opt. Express 20 (2012) 22976. 